\title{
Keynote Lecture 2
}

\author{
S9 \\ Specific Social Skills Training for Patients \\ with Schizophrenia: \\ A Multi-centre Study on the Further Development of IPT
}

\author{
Hans D. Brenner \\ Department of Social and Community Psychiatry, Psychiatric Services of \\ the University of Bern, Bern, Switzerland
}

Social skills training is widely accepted as an additional part of therapy for patients with schizophrenia. As the PORT review states, there is a strong supporting evidence that the patients acquire and maintain social skills. However, the results in the areas of generation, social functioning and psychopathology are still not convincing. New intervention programmes, which additionally include specific cognitive methods and rehabilitation topics that are especially relevant for schizophrenic patients are proposed. Currently there are a few empirical studies available on the efficacy of these therapies. These results are promising and refer to the critical points of the PORT review.

Based on these findings and on our own work on the Integrated Psychological Therapy Programme for Schizophrenic Patients (IPT) we developed three additional cognitive behaviour therapy subprogrammes for the treatment of residential, vocational and recreational functioning.

In a multi-centre-study 120 patients, who have been diagnosed according to DSM-IV criteria to have schizophrenia, have been included. The patients in experimental groups take part in one of the above mentioned specific programmes. The patients in the control group participate in a general social skills training. The duration of the therapy was six months for all groups. A first follow-up assessment was carried out after a period of six months. Social functioning, cognitive abilities and psychopathology of the different groups were compared. Results show that there are significant improvements with respect to dependent variables in the experimental groups. Conclusions will be drawn for improved treatment and rehabilitation approaches for patients with schizophrenia. 